\title{
SVN J \\ Acute cerebrovascular disease following COVID-19: a single center, retrospective, observational study
}

\author{
Yanan Li, ${ }^{1}$ Man Li, ${ }^{1}$ Mengdie Wang, ${ }^{1}$ Yifan Zhou, ${ }^{1}$ Jiang Chang, ${ }^{2}$ Ying Xian, ${ }^{3}$ \\ David Wang (D) , ${ }^{4}$ Ling Mao, ${ }^{1}$ Huijuan Jin, ${ }^{1}$ Bo Hu (D) ${ }^{1}$
}

To cite: Li Y, Li M, Wang M, et al. Acute cerebrovascular disease following COVID-19: a single center, retrospective observational study. Stroke \& Vascular Neurology 2020;5: e000431. doi:10.1136/svn2020-000431

YL, ML, MW, YZ and JC contributed equally.

YL, ML, MW, YZ and JC are joint first authors.

Received 19 May 2020 Revised 5 June 2020 Accepted 12 June 2020 Published Online First 2 July 2020
Check for updates

(c) Author(s) (or their employer(s)) 2020. Re-use permitted under CC BY-NC. No commercial re-use. See rights and permissions. Published by BMJ.

For numbered affiliations see end of article.

Correspondence to Professor Bo Hu; hubo@mail.hust.edu.cn

Professor Huijuan Jin; jinhuijuan1983@163.com

\section{ABSTRACT}

Background and purpose COVID-19 is an infectious disease caused by Severe Acute Respiratory Syndrome Coronavirus 2 (SARS-CoV-2). Apart from respiratory complications, acute cerebrovascular disease (CVD) has been observed in some patients with COVID-19. Therefore, we described the clinical characteristics, laboratory features, treatment and outcomes of CVD complicating SARS-CoV-2 infection.

Materials and methods Demographic and clinical characteristics, laboratory findings, treatments and clinical outcomes were collected and analysed. Clinical characteristics and laboratory findings of patients with COVID-19 with or without new-onset CVD were compared.

Results of 219 patients with COVID-19, 10 (4.6\%) developed acute ischaemic stroke and $1(0.5 \%)$ had intracerebral haemorrhage. COVID-19 with new onset of CVD were significantly older $(75.7 \pm 10.8$ years vs $52.1 \pm 15.3$ years, $p<0.001)$, more likely to present with severe COVID-19 ( $81.8 \%$ vs $39.9 \%, p<0.01)$ and were more likely to have cardiovascular risk factors, including hypertension, diabetes and medical history of CVD (all $p<0.05)$. In addition, they were more likely to have increased inflammatory response and hypercoagulable state as reflected in $\mathrm{C}$ reactive protein $(51.1$ (1.3-127.9) vs $12.1(0.1-212.0) \mathrm{mg} / \mathrm{L}, \mathrm{p}<0.05)$ and D-dimer $(6.9(0.3-$ $20.0)$ vs $0.5(0.1-20.0) \mathrm{mg} / \mathrm{L}, \mathrm{p}<0.001)$. Of 10 patients with ischemic stroke; 6 received antiplatelet treatment with aspirin or clopidogrel; and 3 of them died. The other four patients received anticoagulant treatment with enoxaparin and 2 of them died. As of 24 March 2020, six patients with CVD died (54.5\%).

Conclusion Acute CVD is not uncommon in COVID-19. Our findings suggest that older patients with risk factors are more likely to develop CVD. The development of CVD is an important negative prognostic factor which requires further study to identify optimal management strategy to combat the COVID-19 outbreak.

\section{INTRODUCTION}

Since the first identification of severe acute respiratory syndrome coronavirus (SARS-CoV-2) infection in Wuhan in December $2019,{ }^{1}$ as of 8 April 2020, the number of laboratory-confirmed COVID-19 cases has exceeded 4338658 cases globally, causing over 297119 deaths. ${ }^{12}$ The clinical presentation of patients with COVID-19 varied considerably, ranging from asymptomatic infection to severe pneumonia that may lead to respiratory failure and death. ${ }^{3}$

We recently reported the clinical manifestations and outcomes in 214 patients with COVID-19 infection and found that severe patients had commonly complicated neurological symptoms including consciousness impairment $(14.8 \%)$ and skeletal muscle symptoms (19.3\%). ${ }^{4}$ Previous study has suggested that bacterial and/or viral infection may be a trigger for acute ischaemic stroke, probably related to the prothrombotic effect of the inflammatory response. ${ }^{5}$ To date, there is sparse information regarding acute cerebrovascular disease (CVD) following COVID-19 infection. Accordingly, we reported 11 case series which suffered from SARS-CoV-2 infection and developed a sudden onset of CVDs meanwhile. The clinical characteristics, treatment strategies and outcomes of patients with COVID-19 with and without new-onset CVD are presented here.

\section{METHODS}

This was a single-centre retrospective study. A total of 219 consecutive patients with confirmed COVID-19 were admitted to the Union Hospital of Huazhong University of Science and Technology between 16 January and 19 February, followed up to 24 March 2020. Union Hospital, located in the endemic areas of COVID-19 in Wuhan, Hubei Province, is one of the major tertiary healthcare systems and teaching hospitals in the region and has been designated by the government as a COVID-19 care hospital since the outbreak, responsible for the treatments for SARS-CoV-2 infection. All patients with COVID-19 in this study were diagnosed according to the WHO interim guideline, ${ }^{6}$ had respiratory symptom, were SARS-CoV-2 Reverse Transcription-PCR-PCR positive in throat swab and had viral-like pneumonia on chest $\mathrm{CT}^{7}{ }^{7}$ Part of analysis results of 214 
patients with COVID-19 had been published in our previous paper on JAMA Neurology. ${ }^{4}$ In this study, the analysis results of 219 patients are derived completely from different perspectives and had not been published in any other paper. Verbal consent was obtained from patients or their relatives before the enrolment.

The demographic characteristics, medical history, symptoms, clinical signs, laboratory findings and chest CT scan findings were extracted from electronic medical records. The diagnosis of acute ischaemic or bleeding stroke was confirmed by brain CT and clinical symptoms. All neurological symptoms were reviewed and confirmed by two trained neurologists. The date of disease onset was defined as the day when the symptom was noticed. We dichotomised the patients as severe group and nonsevere group according to the severity of COVID-19 at the time of admission using the American Thoracic Society guidelines for community-acquired pneumonia. ${ }^{89}$ Throat swab samples were collected and placed into a collection tube containing preservation solution for the virus. ${ }^{7}$ SARS-CoV-2 infection was confirmed by real-time RT-PCR assay using a SARS-CoV-2 nucleic acid detection kit according to the manufacturer's protocol (Shanghai BioGerm Medical Technology Co). A total of 219 consecutive patients with confirmed COVID-19 were enrolled. The types of ischaemic stroke were classified by the Trial of Org 10172 in Acute Stroke Treatment (TOAST) classification.

\section{Statistical analysis}

Continuous variables were described as means and SD, or as median and range values between patients with or without new onset of CVD. Categorical variables were expressed as counts and percentages. The Wilcoxon rank-sum test and Maental-Haenszal $\chi^{2}$ test were used to compare characteristics, laboratory findings, treatment and outcomes (with or without acute CVD) of patients with COVID-19 with versus without CVD. All statistical analyses were performed using R V.3.3.0 software, with $\mathrm{p}<0.05$ considered as statistically significant.

\section{RESULTS}

Of 219 patients with confirmed SARS-CoV-2, 11 (5.0\%) developed new onset of CVD following COVID-19 infection. Their demographic and clinical characteristics are shown in table 1 . Of these patients, $10(90.9 \%)$ were diagnosed with ischaemic stroke and $1(9.1 \%)$ had intracerebral haemorrhage. Among the 10 patients with ischaemic stroke, 4 patients' National Institute of Health stroke scale scores was less than 8 points, while 6 patients had scores higher than or equal to 8 points. Representative brain and chest images of patient 1 with ischaemic stroke and patient 11 with intracerebral haemorrhage are shown in figure 1 . The age ranged from 57 to 91 years (median 75 (range 57-91)). Five $(45.5 \%)$ were female. Four $(36.4 \%)$ patients had smoking history and $1(9.1 \%)$ patient had drinking history, respectively. Seven $(63.6 \%)$ patients had increased blood pressure $(\geq 130 / 80 \mathrm{~mm} \mathrm{Hg})$ and 8 $(72.7 \%)$ had elevated blood glucose levels $(>6.1 \mathrm{mmol} / \mathrm{L})$ at diagnosis of CVD. The median durations from first symptoms of SARS-CoV-2 infection to CVD were 10 days (range 1-29). Of the 10 patients with ischaemic stroke, 5 had large-vessel atherosclerosis, 2 had small-vessel occlusion and 3 had cardioembolism type according to TOAST classification. All of these patients were combined with increased inflammatory response and hypercoagulable state. The choice of treatment for ischaemic stroke (antiplatelet/anticoagulant) was determined at the discretion and judgement of the treating team comprehensively reviewing (the TOAST classification, clinical syndrome and laboratory findings).

Of 10 patients with ischaemic stroke, 6 received antiplatelet treatment with aspirin or clopidogrel and 4 received anticoagulant treatment with enoxaparin. As of 24 March 2020, the overall mortality rate was 54.5\% $(6 / 11)$. Among those treated with antiplatelet, three died (50\%) (3/6) as compared with 25\% (1/4) of those treated with anticoagulant. The intracerebral haemorrhage patient died 13 days after stroke.

Table 2 shows the clinical characteristics between patients with COVID-19 with CVD and those without CVD. Patients with COVID-19 with new onset of CVD were significantly older $(75.7 \pm 10.8$ years vs $52.1 \pm 15.3$ years, $\mathrm{p}<0.001)$ and were more likely to present with severe COVID-19 (81.8\% vs 39.9\%, $\mathrm{p}<0.01)$. Moreover, patients with CVD were more likely to have other underlying disorders, including hypertension $(81.8 \%$ vs $22.1 \%$, $\mathrm{p}<0.001)$ and diabetes mellitus ( $54.5 \%$ vs $12.0 \%, \mathrm{p}<0.01)$, which were the common risk factors of CVD.

Table 3 shows the laboratory findings in patients with COVID-19 with or without CVD. Patients with CVD had more increased inflammatory response, including higher white blood cell (median 7.7 (range 3.9-14.8) vs 4.9 (0.1$\left.20.4) \times 10^{9} / \mathrm{L}, \mathrm{p}<0.01\right)$, neutrophil counts $(6.4(0.0-13.8)$ vs $\left.3.0(0.0-18.7) \times 10^{9} / \mathrm{L}, \mathrm{p}<0.01\right)$ and $\mathrm{C}$ reactive protein (CRP) levels (51.1 (1.3-127.9) vs $12.1(0.1-212.0) \mathrm{mg} / \mathrm{L}$, $\mathrm{p}<0.05)$, but lower lymphocyte counts $(0.6(0.3-1.2)$ vs $\left.1.1(0.1-2.6) \times 10^{9} / \mathrm{L}, \mathrm{p}<0.001\right)$, suggesting presence of immunosuppression. Patients with CVD also had higher D-dimer levels $(6.9(0.3-20.0)$ vs $0.5(0.1-20.0) \mathrm{mg} / \mathrm{L}$, $\mathrm{p}<0.001$ ), indicating the hypercoagulable state. In addition, patients with CVD were more likely to have kidney injury, including elevated blood urea nitrogen (7.4 (4.0$43.2)$ vs $4.1(1.5-48.1), \mathrm{p}<0.001)$ and creatinine levels (75.5 (42.7-261.3) vs $68.2(35.9-9435.0), \mathrm{p}<0.05)$.

\section{DISCUSSION}

This is a case series of COVID-19 with new onset of CVD. Eleven patients with COVID-19 developed CVD following infection. Patients with CVD were older and were more likely to have cardiovascular and cerebrovascular risk factors. These findings suggested that elder patients with COVID-19 may be more likely to develop CVD, and more 


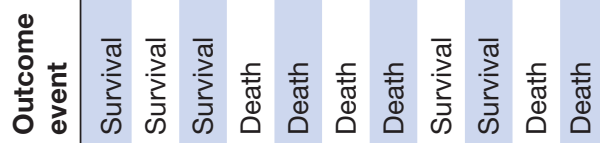

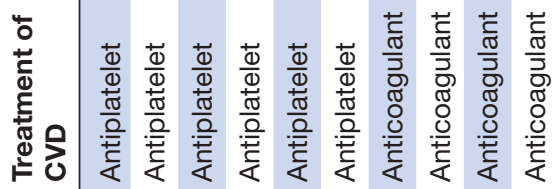

क⿻

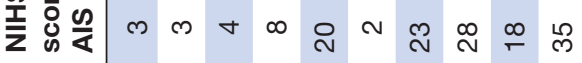

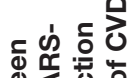

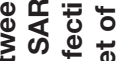

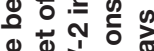

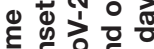

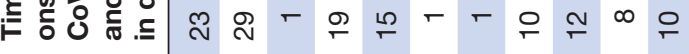

艺

음

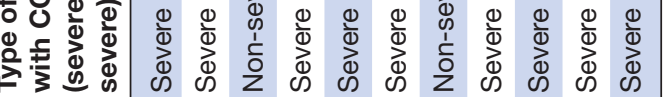

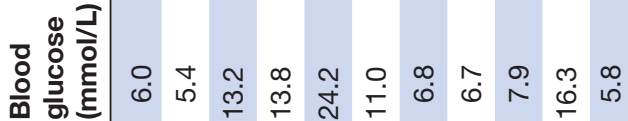

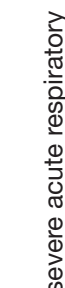

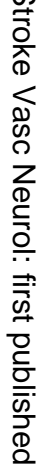

๗

$\vec{\circ}$

$\overrightarrow{\vec{\omega}}$

竞

Nิ

○े

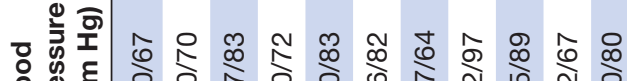

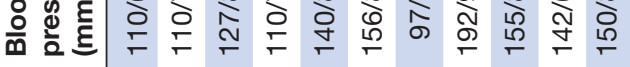

吝

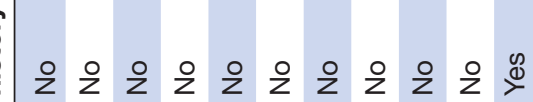

:

政

운은 윤 운 ळँ

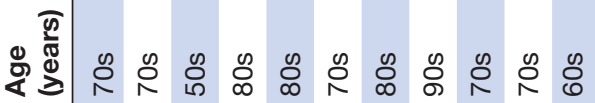

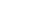

$\frac{10}{20}$

$\frac{\frac{\infty}{0}}{\frac{0}{\omega}}$

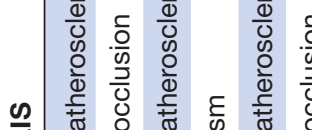

-

¿

\&

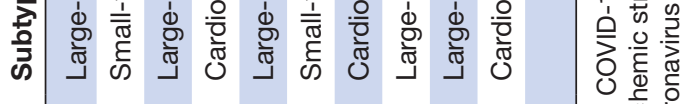

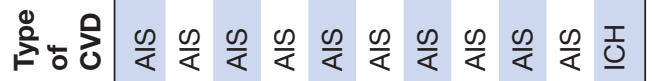

专.

- 
Brain CT
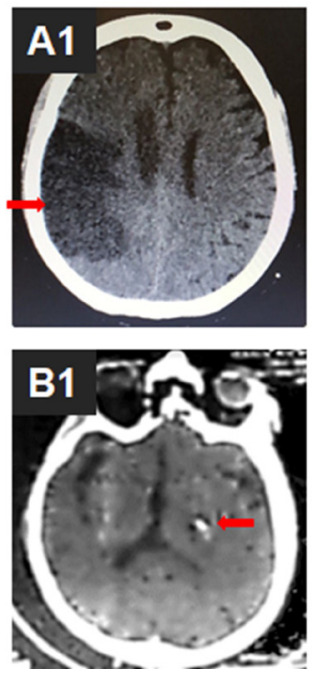

Chest CT
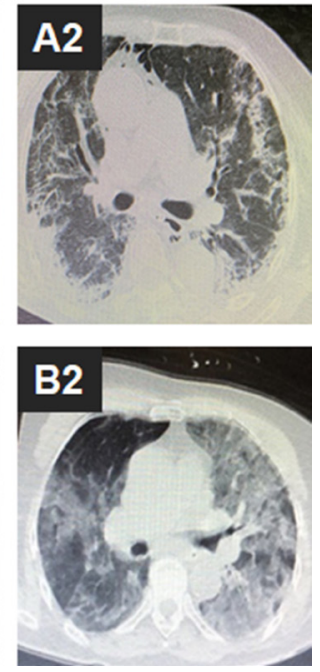

Figure 1 Representative brain and chest images of patients COVID-19 with CVD. (A) Representative brain (A1) and chest (A2) CT images of patient 1 with new onset of ischaemic stroke. The red arrow showed location of infarct in figure A1. (B) Representative brain (B1) and chest (B2) CT images of patient 11 with new onset of intracerebral haemorrhage. CVD, cerebrovascular disease. The red arrow showed location of cerebral hemorrhage in figure B1.

attention should be paid to older patients with cerebrovascular risk factors.

Importantly, 9 out of 11 patients with CVD were patients with SARS-CoV-2 infection, suggesting severe infection may be associated with CVD, especially acute ischaemic stroke. Our past work also showed that severe patients were more likely to develop neurological symptoms. ${ }^{4}$ SARS-CoV-2 has a spike protein surface unit that highly binds to human ACE2 receptor; it may cause endothelial apoptosis and neuronal damage. ${ }^{10}{ }^{11}$ Studies have found that infection with COVID-19 can accelerate the progression of acute stroke. ${ }^{12-14}$ In a total of 4448 patients with COVID-19 in 16 studies, CVD was positively associated with adverse outcome (RR 2.04 (1.43-2.91], $\mathrm{p}<0.001$; i2:77\%). ${ }^{10}$ In an imaging screening of inpatients with COVID-19 infection, ischaemic stroke was found in $0.9 \%$, accompanied by D-dimer peak and increased troponin. ${ }^{15}$ Patients with a history of CVD were 2.5 times more likely to develop severe COVID-19. ${ }^{16}$

Inflammation has been increasingly recognised as a key contributor to the pathophysiology of $\mathrm{CVDs}^{17}$ and involved in the acute intravascular events triggered by the interruption of the blood supply. Several studies have reported the elevated levels of CRPs and D-dimer in patients with the coexistence of COVID-19. ${ }^{13151819} \mathrm{~A}$ study done in China with 191 patients included found that D-dimer greater than $1 \mu \mathrm{g} / \mathrm{mL}$ has an association with mortality in patients of COVID- $19 .{ }^{20}$ Meanwhile, acute inflammation caused by COVID-19 is prone to be followed by a hypercoagulable state due to the increased concentrations of proinflammatory cytokines $^{6-8}$ and serum inflammatory factors (eg, interleukin and CRP) responsible for early molecular events triggered by coagulation abnormalities. ${ }^{21}{ }^{22}$ Compared with the patients without CVD, patients with CVD had a higher blood CRP level. Ten out of 11 patients with both CVD and COVID-19

Table 2 Clinical characteristics of patients with COVID-19 with or without new-onset CVD

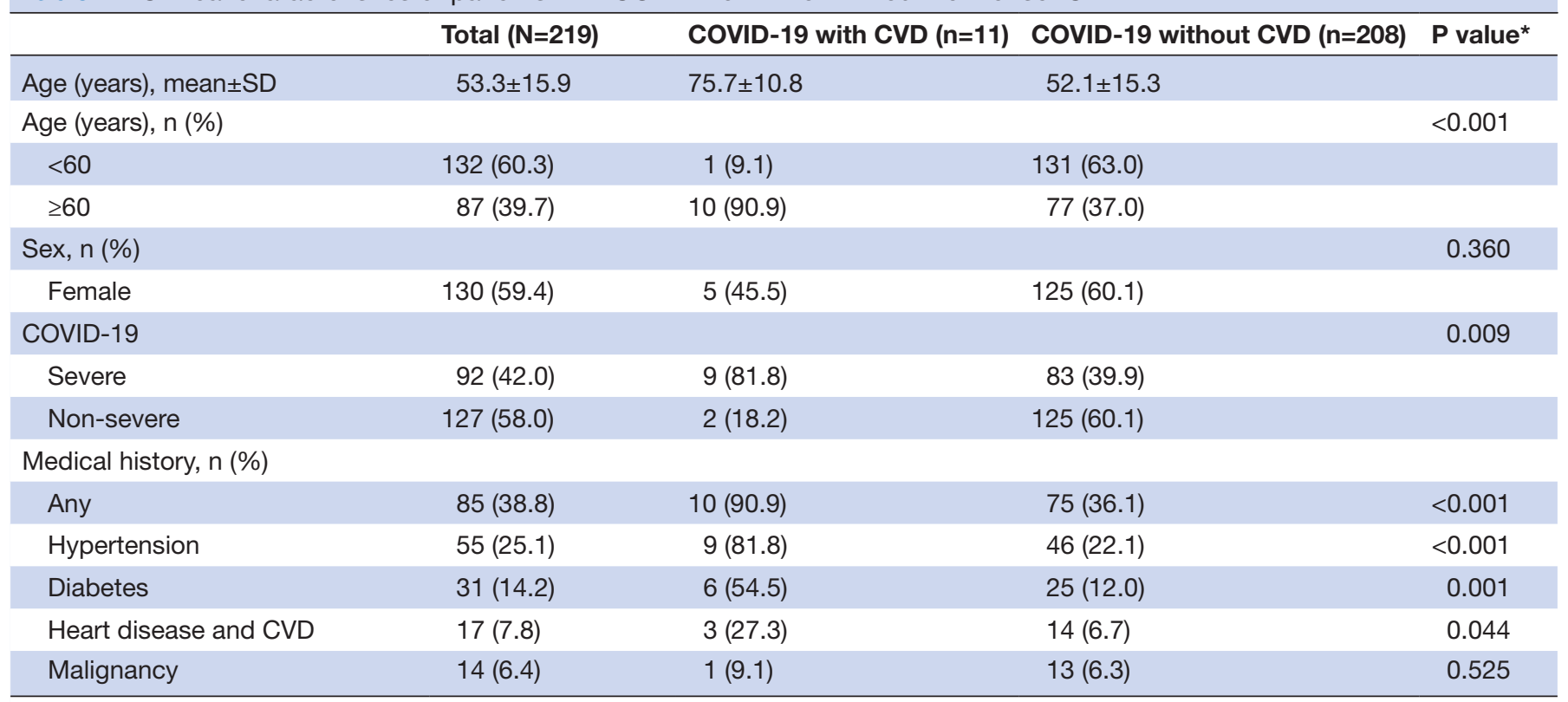

Data are presented as mean \pm SD and $n / N(\%)$.

$P$ values indicate differences between patients with COVID-19 with and without new-onset CVD. $P<0.05$ was considered statistically significant.

${ }^{*}$ Fisher exact test.

CVD, cerebrovascular disease. 
Table 3 Laboratory findings of patients with COVID-19 with or without new-onset CVD

\begin{tabular}{|c|c|c|c|c|}
\hline & $\begin{array}{l}\text { Total } \\
(\mathrm{N}=219)\end{array}$ & $\begin{array}{l}\text { COVID-19 with CVD } \\
(n=11)\end{array}$ & $\begin{array}{l}\text { COVID-19 without CVD } \\
(n=208)\end{array}$ & P value* \\
\hline White blood cell count, $\times 10^{9} / \mathrm{L}$ & $5.0(0.1-20.4)$ & $7.7(3.9-14.8)$ & $4.9(0.1-20.4)$ & 0.002 \\
\hline Lymphocyte count, $\times 10^{9} / \mathrm{L}$ & $1.1(0.1-2.6)$ & $0.6(0.3-1.2)$ & $1.1(0.1-2.6)$ & $<0.001$ \\
\hline Platelet count, $\times 10^{9} / \mathrm{L}$ & $206.0(18.0-583.0)$ & $142.0(90.0-564.0)$ & $211.0(18.0-583.0)$ & 0.035 \\
\hline Alanine aminotransferase (U/L) & $26.0(5.0-1933.0)$ & $24.0(13.0-144.0)$ & $26.5(5.0-1933.0)$ & 0.671 \\
\hline $\begin{array}{l}\text { Aspartate aminotransferase } \\
(\mathrm{U} / \mathrm{L})\end{array}$ & $26.0(8.0-8191.0)$ & $32.0(19.0-271.0)$ & $26.0(8.0-8191 \cdot 0)$ & 0.119 \\
\hline Blood urea nitrogen (mmol/L) & $4.1(1.5-48.1)$ & $7.4(4.0-43.2)$ & $4.1(1.5-48.1)$ & $<0.001$ \\
\hline
\end{tabular}

$P$ values indicate differences between patients with COVID-19 with and without new-onset CVD. $P<0.05$ was considered statistically significant.

*Wilcox non-parameter test.

CVD, cerebrovascular disease.

had extremely high levels of D-dimer (median level of 6.9), by contrast, D-dimer of patients without CVD was at a lower level (median level of 0.5). According to the laboratory test index of blood, patients of COVID-19 with CVD had more severe inflammatory infection and were in a state of high coagulation. The significantly increased inflammatory response could be one of the causes of abnormal blood coagulation function in early stage and could be one of the reasons of onset of CVD.

The average time from SARS-CoV-2 infection to onset of CVD was about 12 days. Neurological injury has been confirmed in the infection of other coronaviruses such as in SARS-CoV and Middle East respiratory syndrome corona virus (MER-CoV) ${ }^{23}{ }^{24} \mathrm{~A}$ study reported that 28 days after onset of SARS-CoV infection, patients experienced central nervous symptoms. ${ }^{25} \mathrm{~A}$ four-patient case report showed patients developed neuromuscular diseases approximately 3 weeks after the onset of SARS. ${ }^{26}$ Another three-patient case report showed brain MRI revealed significant changes characterised by widespread, bilateral hyperintense lesions on T2-weighted imaging within the white matter and subcortical areas of the frontal, temporal and parietal lobes after 25-28 days' onset of MER-CoV ${ }^{23}$ Thus, in early stages, making a diagnosis according to clinical symptoms and lab tests and taking measures for anti-inflammatory treatment as early as possible may arrest the growth of COVID-19. It may be one way to reduce the risk of CVD.

This study has several limitations. First, only 219 patients were studied. It would be better to include more patients from more cities in China or even other countries. Second, all data were abstracted from the electronic medical records, and patients with ischaemic stroke with history of atrial fibrillation were classified into cardioembolism (TOAST classification). The COVID-19 outbreak limited some ancillary examinations such as ultrasonic testing and holter monitoring, which is indispensable for correct TOAST classification. Third, all the 219 consecutive patients with confirmed COVID-19 were symptomatic. If this cohort included asymptomatic cases, it will be more meaningful.

In conclusion, CVD is not uncommon in patients with COVID-19. Patients with CVD were older, had severe infection and were more likely to have cardiovascular and cerebrovascular risk factors. The median time from SARS-CoV-2 infection to onset of CVD was about 10 days. Some patients with COVID-19 with CVD were in a state of high coagulation. Physicians should pay more attention to controlling risk factors for CVDs, such as hypertension, high coagulation state and diabetes mellitus while treating the older or severe patients with COVID-19.

\section{Author affiliations}

${ }^{1}$ Department of Neurology Union Hospital, Tongji Medical College, Huazhong University of Science and Technology, Wuhan, China

${ }^{2}$ Department of Epidemiology and Biostatistics, Key Laboratory for Environment and Health, School of Public Health, Tongji Medical College, Huazhong University of Science and Technology, Wuhan, China

${ }^{3}$ Duke Clinical Research Institute and Department of Neurology, Duke University Medical Center, Durham, North Carolina, USA

${ }^{4}$ Neurovascular Division, Department of Neurology, Barrow Neurological Institute/ Saint Joseph Hospital Medical Center, Phoenix, Arizona, USA

Contributors Concept and design: $\mathrm{BH}, \mathrm{YL}$; acquisition, analysis or interpretation of data: JC, $\mathrm{HJ}, \mathrm{ML}, \mathrm{MW}, \mathrm{YZ}, \mathrm{YX}$; drafting of the manuscript: $\mathrm{BH}, \mathrm{YL}, \mathrm{ML}, \mathrm{HJ}, \mathrm{MW}$, JC, YZ, YX; critical revision of the manuscript for important intellectual content: $\mathrm{BH}$; statistical analysis: JC, $\mathrm{YL}$; obtained funding: $\mathrm{BH}$; administrative, technical or material support: $\mathrm{BH}, \mathrm{YL}, \mathrm{HJ}, \mathrm{ML}, \mathrm{MW}, \mathrm{YZ}$, YX; supervision: $\mathrm{BH}$.

Funding This study was funded by the National Key Research and Development Program of China (number 2018YFC1312200 to BH) and the National Natural Science Foundation of China (number 81820108010 to BH).

Competing interests None declared.

Patient consent for publication Not required.

Provenance and peer review Not commissioned; externally peer reviewed. 
Data availability statement Data are available upon reasonable request. The data that support the findings of this study are available from the corresponding author, BH (Department of Neurology, Union Hospital, Tongji Medical College, Huazhong University of Science and Technology, Wuhan, China. hubo@mail.hust.edu.cn), upon reasonable request.

Open access This is an open access article distributed in accordance with the Creative Commons Attribution Non Commercial (CC BY-NC 4.0) license, which permits others to distribute, remix, adapt, build upon this work non-commercially, and license their derivative works on different terms, provided the original work is properly cited, appropriate credit is given, any changes made indicated, and the use is non-commercial. See: http://creativecommons.org/licenses/by-nc/4.0/.

ORCID iDs

David Wang http://orcid.org/0000-0003-2277-4608

Bo Hu http://orcid.org/0000-0003-1462-8854

\section{REFERENCES}

1 China NHCotPsRo. Update on the outbreak of coronavirus disease 2019 as of 24:00 on April 7, 2020. Available: http://www.nhc.gov. cn/xcs/yqtb/202004/5e2b6f0bd47d48559582242e3878447d.shtml [Accessed 8 Apr 2020].

2 WHO. Coronavirus disease 2019 (COVID-19) situation report-78, 2020. Available: https://www.who.int/docs/default-source/ coronaviruse/situation-reports/20200407-sitrep-78-covid-19.pdf? sfvrsn=bc43e1b 2 [Accessed 8 Apr 2020].

3 Wang D, Hu B, Hu C, et al. Clinical characteristics of 138 hospitalized patients with 2019 novel coronavirus-infected pneumonia in Wuhan, China. JAMA 2020;323:1061.

4 Mao L, Wang M, Chen S, et al. Neurological manifestations of hospitalized patients with COVID-19 in Wuhan, China: a retrospective case series study. SSRN Journal 2020.

5 Grau AJ, Buggle F, Becher $\mathrm{H}$, et al. Recent bacterial and viral infection is a risk factor for cerebrovascular ischemia: clinical and biochemical studies. Neurology 1998;50:196-203.

6 WHO. Clinical management of severe acute respiratory infection when novel coronavirus (nCoV) infection is suspected, 2020. Available: https://www.who.int/publications-detail/clinicalmanagement-of-severe-acute-respiratory-infection-when-novelcoronavirus-(ncov)-infection-is-suspected [Accessed 28 Feb 2020].

7 Huang C, Wang Y, Li X, et al. Clinical features of patients infected with 2019 novel coronavirus in Wuhan, China. Lancet 2020;395:497-506.

8 Metlay JP, Waterer GW, Long AC, et al. Diagnosis and treatment of adults with community-acquired pneumonia. An official clinical practice guideline of the American thoracic Society and infectious diseases Society of America. Am J Respir Crit Care Med 2019;200:e45-67.
9 W-j G, Z-y N, Hu Y, et al. Clinical characteristics of coronavirus disease 2019 in China. New Engl J Med 2020.

10 Pranata R, Huang I, Lim MA, et al. Impact of cerebrovascular and cardiovascular diseases on mortality and severity of COVID-19 systematic review, meta-analysis, and meta-regression. J Stroke Cerebrovasc Dis 2020;104949:104949.

11 Vonck K, Garrez I, De Herdt V, et al. Neurological manifestations and neuro-invasive mechanisms of the severe acute respiratory syndrome coronavirus type 2. Eur J Neurol 2020;14. doi:10.1111/ ene.14329. [Epub ahead of print: 16 May 2020].

12 Goldberg MF, Goldberg MF, Cerejo R, et al. Cerebrovascular disease in COVID-19. AJNR Am J Neuroradiol 2020. doi:10.3174/ajnr.A6588. [Epub ahead of print: 14 May 2020].

13 Tunç A, Ünlübaș Y, Alemdar M, et al. Coexistence of COVID-19 and acute ischemic stroke report of four cases. J Clin Neurosci 2020. doi:10.1016/j.jocn.2020.05.018. [Epub ahead of print: 06 May 2020].

14 Avula A, Nalleballe K, Narula N, et al. COVID-19 presenting as stroke. Brain Behav Immun 2020. doi:10.1016/j.bbi.2020.04.077. [Epub ahead of print: 28 Apr 2020].

15 Yaghi S, Ishida K, Torres J, et al. SARS2-CoV-2 and stroke in a New York healthcare system. Stroke 2020;24.

16 Aggarwal G, Lippi G, Michael Henry B. Cerebrovascular disease is associated with an increased disease severity in patients with coronavirus disease 2019 (COVID-19): a pooled analysis of published literature. Int J Stroke 2020;15:385-9.

17 ladecola $\mathrm{C}$, Anrather J. The immunology of stroke: from mechanisms to translation. Nat Med 2011;17:796-808.

18 Mao L, Jin H, Wang M, et al. Neurologic manifestations of hospitalized patients with coronavirus disease 2019 in Wuhan, China. JAMA Neurol 2020:e201127.

19 Valderrama EV, Humbert K, Lord A, et al. Severe acute respiratory syndrome coronavirus 2 infection and ischemic stroke. Stroke 2020:STROKEAHA120030153.

20 Zhou F, Yu T, Du R, et al. Clinical course and risk factors for mortality of adult inpatients with COVID-19 in Wuhan, China: a retrospective cohort study. Lancet 2020;395:1054-62.

21 Ding P, Zhang S, Yu M, et al. II-17A promotes the formation of deep vein thrombosis in a mouse model. Int Immunopharmacol 2018;57:132-8

22 Horvei LD, Grimnes G, Hindberg K, et al. C-reactive protein, obesity, and the risk of arterial and venous thrombosis. J Thromb Haemost 2016;14:1561-71.

23 Arabi YM, Harthi A, Hussein J, et al. Severe neurologic syndrome associated with middle east respiratory syndrome corona virus (MERS-CoV). Infection 2015;43:495-501.

24 Umapathi T, Kor AC, Venketasubramanian N, et al. Large artery ischaemic stroke in severe acute respiratory syndrome (SARS). $J$ Neurol 2004;251:1227-31.

$25 \mathrm{Xu}$ J, Zhong S, Liu J, et al. Detection of severe acute respiratory syndrome coronavirus in the brain: potential role of the chemokine Mig in pathogenesis. Clin Infect Dis 2005;41:1089-96.

26 Tsai L-K, Hsieh S-T, Chao C-C, et al. Neuromuscular disorders in severe acute respiratory syndrome. Arch Neurol 2004;61:1669-73. 